\title{
Strong convergence for total asymptotically pseudocontractive semigroups in Banach spaces
}

Xiongrui Wang* and Jing Quan

\section{"Correspondence:} wxr888x@163.com Department of Mathematics, Yibin University, Yibin, Sichuan 644007, China

\begin{abstract}
In this article, the demiclosedness principle for total asymptotically pseudocontractions in Banach spaces is established. The strong convergence to a common fixed point of total asymptotically pseudocontractive semigroups in Banach spaces is established based on the demiclosedness principle, the generalized projective operator, and the hybrid method. The main results presented in this article extend and improve the corresponding results of many authors.
\end{abstract}

MSC: 47H05; 47H09; 49M05

Keywords: strong convergence; total asymptotically pseudocontractive semigroups; common fixed point

\section{Introduction}

Throughout this article, we assume that $E$ is a real Banach space with the norm $\|\cdot\|, E^{*}$ is the dual space of $E ;\langle\cdot, \cdot\rangle$ is the duality pairing between $E$ and $E^{*} ; C$ is a nonempty closed convex subset of $E ; \mathbb{N}$ and $\mathbb{R}^{+}$denote the natural number set and the set of nonnegative real numbers, respectively. The mapping $J: E \rightarrow 2^{E^{*}}$ defined by

$$
J(x)=\left\{f^{*} \in E^{*}:\left\langle x, f^{*}\right\rangle=\|x\|^{2} ;\left\|f^{*}\right\|=\|x\|, x \in E\right\}
$$

is called the normalized duality mapping. Let $T: C \rightarrow C$ be a nonlinear mapping; $F(T)$ denotes the set of fixed points of the mapping $T$. We use ' $\rightarrow$ ' to stand for strong convergence and ' $\rightarrow$ ' for weak convergence.

As is well known, fixed theory has always been kept a watchful eye on in nonlinear analysis, such as [1] proved Krasnoselskii's fixed point theorem for general classes of maps, [2] established strong convergence theorems for fixed point problems and generalized equilibrium problems of three relatively quasi-nonexpansive mappings in a Banach space. Common fixed points of semigroups have also been discussed; see [3-5]. The construction of fixed points of pseudocontractive mappings (asymptotically pseudocontractive mappings), and of common fixed points of pseudocontractive semigroups (asymptotically pseudocontractive semigroups) is an important problem in the theory of pseudocontractive mappings. Iterative approximation of fixed points or common fixed points for asymptotically pseudocontractive mappings, pseudocontractive semigroups, asymptotically pseudocontractive semigroups in Hilbert or Banach spaces has been studied exten- 
sively by many authors; see for example [4, 6-12]. Alber et al. [13] introduced a more general class of asymptotically nonexpansive mappings called total asymptotically nonexpansive mappings and studied the methods of approximation of fixed points. Qin et al. [14] have introduced total asymptotically pseudocontractive mappings and proved a weak convergence theorem of fixed points for total asymptotically pseudocontractive mappings in Hilbert spaces.

The pseudocontractive semigroup and asymptotically pseudocontractive semigroup are defined as follows.

Definition 1.1 [4] One-parameter family $\mathbf{T}:=\{T(t): t \geq 0\}$ of mappings from $C$ into itself is said to be a pseudocontractive semigroup on $C$, if the following conditions are satisfied:

(a)

$$
T(0) x=x \text { for each } x \in C \text {; }
$$

(b)

$$
T(t+s) x=T(s) T(t) \text { for any } t, s \in \mathbb{R}^{+} \text {and } x \in C ;
$$

(c)

For any $x \in C$, the mapping $t \rightarrow T(t) x$ is continuous;

(d)

For all $x, y \in C$, there exists $j(x-y) \in J(x-y)$ such that

$$
\langle T(t) x-T(t) y, j(x-y)\rangle \leq\|x-y\|^{2}, \quad \text { for any } t \geq 0
$$

holds.

Definition 1.2 One-parameter family $\mathbf{T}:=\{T(t): t \geq 0\}$ of mappings from $C$ into itself is said to be an asymptotically pseudocontractive semigroup on $C$, if the conditions (a), (b), (c) in Definition 1.1 and the following condition (e) are satisfied:

(e) For all $x, y \in C, t \geq 0$, there exists a sequence $\left\{k_{n}\right\}$ of positive real numbers with $k_{n} \geq 1, \lim _{n \rightarrow+\infty} k_{n}=1$, and there exists $j(x-y) \in J(x-y)$ such that

$$
\left\langle T^{n}(t) x-T^{n}(t) y, j(x-y)\right\rangle \leq k_{n}\|x-y\|^{2}, \quad n \in \mathbb{N}
$$

holds.

The purpose of this article is to introduce the concept of a total asymptotically pseudocontractive semigroup and to use the hybrid method for total asymptotically pseudocontractive semigroups to get the strong convergence in Banach spaces. The results presented in the article improve and extend the corresponding results of many authors.

\section{Preliminaries}

In the sequel, we assume that $E$ is a smooth, strictly convex, and reflexive Banach space and $C$ is a nonempty closed convex subset of $E$. We use $\phi: E \times E \rightarrow \mathbb{R}^{+}$to denote the Lyapunov functional defined by

$$
\phi(x, y)=\|x\|^{2}-2\langle x, J y\rangle+\|y\|^{2}, \quad \forall x, y \in E .
$$

It is obvious that the following conditions hold, $\forall x, y, z \in E$ : 
1. $(\|x\|-\|y\|)^{2} \leq \phi(x, y) \leq(\|x\|+\|y\|)^{2}$;

2. $\phi(x, y)=\phi(x, z)+\phi(z, y)+2\langle x-z, J z-J x\rangle$;

3. $\phi(x, y)=\langle x, J x-J y\rangle+\langle y-x, J y\rangle \leq\|x\|\|J x-J y\|+\|y-x\|\|y\|$.

Following Alber [15], the generalized projection $\Pi_{C} x: E \rightarrow C$ is defined by

$$
\Pi_{C} x=\arg \inf _{y \in C} \phi(y, x), \quad \forall x \in E .
$$

In order to prove the results of this paper, we shall need the following lemmas.

Lemma 2.1 [16] Let E be a uniformly convex and smooth Banach space and let $\left\{x_{n}\right\}$ and $\left\{y_{n}\right\}$ be two sequences of $E$. If $\phi\left(x_{n}, y_{n}\right) \rightarrow 0$ and either $\left\{x_{n}\right\}$ or $\left\{y_{n}\right\}$ is bounded, then $\| x_{n}-$ $y_{n} \| \rightarrow 0$.

Lemma 2.2 [15] Let E be a smooth, strictly convex, and reflexive Banach space and C be a nonempty closed convex subset of $E$. Then the following conditions hold:

(i) $\phi\left(x, \Pi_{C} y\right)+\phi\left(\Pi_{C} y, y\right) \leq \phi(x, y)$ for all $x \in C, y \in E$;

(ii) If $x \in E$ and $z \in C$, then $z=\Pi_{C} x \Leftrightarrow\langle z-y$,Jx-Jz> $\geq 0, \forall y \in C$;

(iii) For $x, y \in E, \phi(x, y)=0$ if and only if $x=y$.

Qin[14] introduced the class of total asymptotically pseudocontractive mappings in Hilbert spaces and established a weak convergence theorem of fixed points. Now, we give the definition of total asymptotically pseudocontractive mappings in a Banach space.

Definition 2.1 The mapping $T$ from $C$ into itself is said to be total asymptotically pseudocontractive on $C$ if, for all $x, y \in C, t \geq 0$, there exist sequences $\left\{\mu_{n}\right\},\left\{v_{n}\right\}$ with $\mu_{n}, v_{n} \rightarrow 0$ as $n \rightarrow \infty$ and strictly increasing continuous functions $\psi: \mathbb{R}^{+} \rightarrow \mathbb{R}^{+}$with $\psi(0)=0$, and there exists $j(x-y) \in J(x-y)$ such that

$$
\left\langle T^{n} x-T^{n} y, j(x-y)\right\rangle \leq\|x-y\|^{2}+\mu_{n} \psi(\|x-y\|)+v_{n}, \quad n \in \mathbb{N}
$$

holds.

Remark 2.1 If $\psi(\lambda)=\lambda^{2}$, then $\left\langle T^{n} x-T^{n} y, j(x-y)\right\rangle \leq\|x-y\|^{2}+\mu_{n} \psi(\|x-y\|)+v_{n}$ reduces to $\left\langle T^{n} x-T^{n} y, j(x-y)\right\rangle \leq\left(1+\mu_{n}\right)\|x-y\|^{2}+v_{n}$, so the total asymptotically pseudocontractive mappings include asymptotically pseudocontractive mappings. If $\mu_{n}=0, v_{n}=0$ for all $n \geq 1$, then total asymptotically pseudocontractive mappings coincide with the class of pseudocontractive mappings.

Yang [17] has studied total asymptotically strict pseudocontractive semigroups. We now introduce the following semigroup mappings.

Definition 2.2 (1) One-parameter family $\mathbf{T}:=\{T(t): t \geq 0\}$ of mappings from $C$ into itself is said to be a total asymptotically pseudocontractive semigroup on $C$ if the conditions (a), (b), (c) in Definition 1.1 and the following condition (f) are satisfied:

(f) For all $x, y \in C, t \geq 0$, there exist sequences $\left\{\mu_{n}\right\},\left\{v_{n}\right\}$ with $\mu_{n}, v_{n} \rightarrow 0$ as $n \rightarrow \infty$ and strictly increasing continuous functions $\psi: \mathbb{R}^{+} \rightarrow \mathbb{R}^{+}$with $\psi(0)=0$, for all 
$x, y, \in C, t \geq 0$, there exists $j(x-y) \in J(x-y)$ such that

$$
\left\langle T^{n}(t) x-T^{n}(t) y, j(x-y)\right\rangle \leq\|x-y\|^{2}+\mu_{n} \psi(\|x-y\|)+v_{n}, \quad n \in \mathbb{N}
$$

holds.

(2) A total asymptotically pseudocontractive semigroup $\mathbf{T}$ is said to be uniformly Lipschitzian if there exists a bounded measurable function $L:[0, \infty) \rightarrow(0,+\infty)$ such that

$$
\left\|T^{(n)}(t) x-T^{(n)}(t) y\right\| \leq L(t)\|x-y\|, \quad \forall x, y \in C, t \geq 0, n \in \mathbb{N} .
$$

In this article, we let $L=\sup _{t \geq 0} L(t)<\infty$.

\section{Main results}

Theorem 3.1 (Demiclosedness principle) Let E be a reflexive smooth Banach space with a weakly sequential continuous duality mapping $J$ and $C$ be a nonempty bounded and closed convex subset of E. Let $\mathbf{T}:=\{T(t): t \geq 0\}$ be a uniformly L-Lipschitzian and total asymptotically pseudocontractive semigroup from $C$ into itself defined by Definition 2.2. Suppose there exists $M^{*}>0$ such that $\psi\left(\xi_{n}\right) \leq M^{*} \xi_{n}$. Then $I-T(t)$ is demiclosed at zero, where I is the identical mapping.

Proof Assume that $\left\{x_{n}\right\} \subset C$, with $x_{n} \rightarrow x$ and $x_{n}-T(t) x_{n} \rightarrow 0$ as $n \rightarrow \infty$. We want to prove $x \in C$ and $x=T(t) x$. Since $C$ is a closed convex subset of $E, C$ is weakly closed. So, $x \in C$. In the following, we prove $x=T(t) x$.

Now, we choose $\alpha \in\left(0, \frac{1}{1+L}\right)$, and let $y_{m}=(1-\alpha) x+\alpha T^{m}(t) x$ for $m \geq 1$. Because $T(t)$ is uniformly $L$-Lipschitzian, we have

$$
\begin{aligned}
\| x_{n}- & T^{m}(t) x_{n} \| \\
\leq & \left\|x_{n}-T(t) x_{n}\right\|+\left\|T(t) x_{n}-T^{2}(t) x_{n}\right\|+\cdots \\
& \quad+\left\|T^{m-1}(t) x_{n}-T^{m}(t) x_{n}\right\| \leq m L\left\|x_{n}-T(t) x_{n}\right\| \rightarrow 0, \quad \text { as } n \rightarrow \infty .
\end{aligned}
$$

Since $T(t)$ is total asymptotically pseudocontractive, we have

$$
\begin{aligned}
\left\langle J\left(x-y_{m}\right),\left(I-T^{m}(t)\right) y_{m}\right\rangle & \\
= & \left\langle J\left(x-y_{m}\right)-J\left(x_{n}-y_{m}\right),\left(I-T^{m}(t)\right) y_{m}\right\rangle+\left\langle J\left(x_{n}-y_{m}\right),\left(I-T^{m}(t)\right) y_{m}\right\rangle \\
= & \left\langle J\left(x-y_{m}\right)-J\left(x_{n}-y_{m}\right),\left(I-T^{m}(t)\right) y_{m}\right\rangle+\left\langle J\left(x_{n}-y_{m}\right),\left(I-T^{m}(t)\right) x_{n}\right\rangle \\
& \quad+\left\langle J\left(x_{n}-y_{m}\right),\left(I-T^{m}(t)\right) y_{m}-\left(I-T^{m}(t)\right) x_{n}\right\rangle \\
\leq & \left\langle J\left(x-y_{m}\right)-J\left(x_{n}-y_{m}\right),\left(I-T^{m}(t)\right) y_{m}\right\rangle+\left\langle J\left(x_{n}-y_{m}\right),\left(I-T^{m}(t)\right) x_{n}\right\rangle \\
& \quad+\mu_{m} \psi\left(\left\|x_{n}-y_{m}\right\|\right)+v_{m} .
\end{aligned}
$$

By assumption $x_{n} \rightarrow x, x_{n}-T(t) x_{n} \rightarrow 0$ and $\left\|x_{n}-T^{m}(t) x_{n}\right\| \rightarrow 0$ as $n \rightarrow \infty, J$ is a weakly sequential continuous duality mapping, we have

$$
\begin{aligned}
\left\langle J\left(x-y_{m}\right),\left(I-T^{m}(t)\right) y_{m}\right\rangle & \leq \mu_{m} \psi\left(\left\|x_{n}-y_{m}\right\|\right)+v_{m} \\
& \leq \mu_{m} M^{*}\left\|x_{n}-y_{m}\right\|+v_{m} \leq \mu_{m} M^{*}(\operatorname{diam} C)+v_{m} .
\end{aligned}
$$


By the $L$-Lipschitz of $T(t)$ and the definition of $y_{m}$, we have

$$
\begin{aligned}
& \left\langle J\left(x-y_{m}\right),\left(I-T^{m}(t)\right) x-\left(I-T^{m}(t)\right) y_{m}\right\rangle \\
& \quad \leq(1+L)\left\|x-y_{m}\right\|^{2} \leq(1+L) \alpha^{2}\left\|x-T^{m}(t) x\right\|^{2} .
\end{aligned}
$$

Thus,

$$
\begin{aligned}
\| x & -T^{m}(t) x \|^{2} \\
& =\left\langle J\left(x-T^{m}(t) x\right), x-T^{m}(t) x\right\rangle=\frac{1}{\alpha}\left\langle J\left(x-y_{m}\right), x-T^{m}(t) x\right\rangle \\
& =\frac{1}{\alpha}\left\langle J\left(x-y_{m}\right), x-T^{m}(t) x-\left(y_{m}-T^{m}(t) y_{m}\right)\right\rangle+\frac{1}{\alpha}\left\langle J\left(x-y_{m}\right),\left(y_{m}-T^{m}(t) y_{m}\right)\right\rangle \\
& \leq \alpha(1+L)\left\|x-T^{m}(t) x\right\|^{2}+\frac{1}{\alpha}\left\langle J\left(x-y_{m}\right),\left(y_{m}-T^{m}(t) y_{m}\right)\right\rangle \\
& \leq \alpha(1+L)\left\|x-T^{m}(t) x\right\|^{2}+\frac{1}{\alpha}\left(\mu_{m} M^{*}(\operatorname{diam} C)+v_{m}\right),
\end{aligned}
$$

which implies that

$$
\alpha[1-\alpha(1+L)]\left\|x-T^{m}(t) x\right\|^{2} \leq \mu_{m} M^{*}(\operatorname{diam} C)+v_{m}, \quad \forall m \in \mathbb{N} .
$$

When $m \rightarrow \infty, \mu_{m}, v_{m} \rightarrow 0$, so we have $\left\|x-T^{m}(t) x\right\| \rightarrow 0, m \rightarrow \infty$, i.e., $T^{m}(t) x \rightarrow x$, $m \rightarrow \infty$, so $T^{m+1}(t) x \rightarrow T(t) x, m \rightarrow \infty$. By the continuity of $T(t)$, we have $T(t) x=x$.

Theorem 3.2 Let E be a real uniformly convex and uniformly smooth Banach space with a weakly sequential continuous convex duality mapping J and C be a nonempty closed convex subset of $E$. Let $\mathbf{T}:=\{T(t): t \geq 0\}$ be a total asymptotically pseudocontractive semigroup of mappings from $C$ into itself defined by Definition 2.2. Suppose there exists $M^{*}>0$ such that $\psi\left(\xi_{n}\right) \leq M^{*} \xi_{n}$. Suppose $\mathbf{T}:=\{T(t): t \geq 0\}$ are closed, convex, uniformly L-Lipschitz and $F(T):=\bigcap_{t \geq 0} F(T(t)) \neq \emptyset$. Let $\alpha_{n}$ be a sequence in $[a, b]$, where $a, b \in\left(0, \frac{1}{1+L}\right)$. Let $x_{n}$ be a sequence generated by

$$
\left\{\begin{aligned}
x_{1}=x \in C, & \\
y_{n}= & \left(1-\alpha_{n}\right) x_{n}+\alpha_{n} T^{n}(t) x_{n}, \\
H_{n}= & \left\{z \in C_{n}: \alpha_{n}\left[1-(1+L) \alpha_{n}\right]\left\|x_{n}-T^{n}(t) x_{n}\right\|^{2}\right. \\
& \left.\leq\left\langle J\left(x_{n}-y_{n}\right)-J\left(z-y_{n}\right),\left(y_{n}-T^{n}(t) y_{n}\right)\right\rangle+\zeta_{n}\right\}, \\
W_{n}= & \left\{z \in C:\left\langle x_{n}-z, J x-J x_{n}\right\rangle\right\} \geq 0, \\
x_{n+1}= & P_{H_{n} \cap W_{n}} x, \quad \forall n \in \mathbb{N},
\end{aligned}\right.
$$

where $\zeta_{n}=\mu_{n} M^{*}($ diam $C)+v_{n}$, then the iterative sequence $\left\{x_{n}\right\}$ converges strongly to a common fixed point $x^{*} \in F(T)$ in $C$.

Proof We divide the proof into six steps.

(I) $P_{F(T)} x$ is well defined for every $x \in C$. Since $\mathbf{T}:=\{T(t): t \geq 0\}$ is uniform $L$-Lipschitzian continuous and convex, so $F(T)$ is closed and convex. Moreover, $F(T)$ is nonempty, therefore, $P_{F(T)} x$ is well defined for every $x \in C$. 
(II) We prove that $H_{n}$ and $W_{n}$ are closed and convex for all $n \in \mathbb{N}$.

From the definitions of $W_{n}$ and $H_{n}$, it is obvious that $W_{n}$ is closed and convex and $H_{n}$ is closed for each $n \in \mathbb{N}$. $H_{n}$ is convex for each $n \in \mathbb{N}$, which follows from the convexity of $J$.

(III) We prove $F(T) \subset H_{n} \cap W_{n}$ for each $n \in \mathbb{N}$.

We first show $F(T) \subset H_{n}$. Let $p \in F(T), n \in \mathbb{N}$. By (7), and the uniform $L$-Lipschitz continuity of $T(t)$ and the total asymptotical pseudocontractiveness of $T(t)$, we have

$$
\begin{aligned}
\| x_{n}- & T^{n}(t) x_{n} \|^{2} \\
= & \left\langle J\left(x_{n}-T^{n}(t) x_{n}\right), x_{n}-T^{n}(t) x_{n}\right\rangle \\
= & \frac{1}{\alpha_{n}}\left\langle J\left(x_{n}-y_{n}\right), x_{n}-T^{n}(t) x_{n}\right\rangle \\
= & \frac{1}{\alpha_{n}}\left\langle J\left(x_{n}-y_{n}\right), x_{n}-T^{n}(t) x_{n}-\left(y_{n}-T^{n}(t) y_{n}\right)\right\rangle \\
& +\frac{1}{\alpha_{n}}\left\langle J\left(x_{n}-y_{n}\right),\left(y_{n}-T^{n}(t) y_{n}\right)\right\rangle \\
\leq & \alpha_{n}(1+L)\left\|x-T^{n}(t) x\right\|^{2}+\frac{1}{\alpha_{n}}\left\langle J\left(x_{n}-y_{n}\right)-J\left(p-y_{n}\right),\left(y_{n}-T^{n}(t) y_{n}\right)\right\rangle \\
& +\frac{1}{\alpha_{n}}\left\langle J\left(p-y_{n}\right),\left(y_{n}-T^{n}(t) y_{n}\right)-\left(p-T^{n}(t) p\right)\right\rangle \\
\leq & \alpha_{n}(1+L)\left\|x_{n}-T^{n}(t) x_{n}\right\|^{2}+\frac{1}{\alpha_{n}}\left\langle J\left(x_{n}-y_{n}\right)-J\left(p-y_{n}\right),\left(y_{n}-T^{n}(t) y_{n}\right)\right\rangle \\
& +\frac{1}{\alpha_{n}}\left(\mu_{n} M^{*}(\operatorname{diam} C)+v_{n}\right) .
\end{aligned}
$$

This implies that

$$
\begin{aligned}
\alpha_{n}[1 & \left.-\alpha_{n}(1+L)\right]\left\|x_{n}-T^{n}(t) x_{n}\right\|^{2} \\
\leq & \left\langle J\left(x_{n}-y_{n}\right)-J\left(p-y_{n}\right),\left(y_{n}-T^{n}(t) y_{n}\right)\right\rangle \\
& +\left(\mu_{n} M^{*}(\operatorname{diam} C)+v_{n}\right), \quad \forall n \in \mathbb{N} .
\end{aligned}
$$

This shows that $p \in H_{n}$ for all $n \in \mathbb{N}$. This proves that $F(T) \subset H_{n}$ for all $n \in \mathbb{N}$. Next, we prove $F(T) \subset W_{n}$ for all $n \in \mathbb{N}$. By induction, for $n=0$, we have $F(T) \subset C=W_{0}$. Assume that $F(T) \subset W_{n}$. Since $x_{n+1}$ is the projection of $x$ onto $H_{n} \cap W_{n}$, by Lemma 2.2, we have

$$
\left\langle x_{n+1}-z, J\left(x-x_{n+1}\right)\right\rangle \geq 0
$$

for any $z \in H_{n} \cap W_{n}$. This with the assumption $F(T) \subset H_{n} \cap W_{n}, u \in F(T)$ and the definition of $W_{N+1}$ leads to $F(T) \subset H_{n} \cap W_{n}, \forall n \in \mathbb{N}$.

(IV) We prove that $\left\|x_{n+1}-x_{n}\right\| \rightarrow 0$ as $n \rightarrow \infty$.

From (7) and Lemma 2.2, we have $x_{n}=P_{w_{n}} x$, this shows, for any $z \in W_{n}$ and $p \in F(T) \subset$ $w_{n}$, we get

$$
\phi\left(x_{n}, x\right) \leq \phi\left(x_{n+1}, x\right) \quad \text { and } \quad \phi\left(x_{n}, x\right) \leq \phi(p, x)
$$


for all $n \in \mathbb{N}$. So, we have $\lim _{n \rightarrow \infty} \phi\left(x_{n}, x\right)$ and $\left\{x_{n}\right\}$ is bounded. So, by Lemma 2.2, we have

$$
\phi\left(x_{n+1}, x_{n}\right) \leq \phi\left(x_{n+1}, x\right)-\phi\left(x_{n}, x\right) \rightarrow 0
$$

as $n \rightarrow \infty$. By Lemma 2.1, we get $\left\|x_{n+1}-x_{n}\right\| \rightarrow 0$ as $n \rightarrow \infty$.

(V) Now, we prove $\left\|x_{n}-T(t) x_{n}\right\| \rightarrow 0$ as $n \rightarrow \infty$ for all $t \geq 0$.

It follows from $\left\|x_{n+1}-x_{n}\right\| \rightarrow 0$ as $n \rightarrow \infty, x_{n+1} \subset H_{n},\left\{y_{n}\right\}$ is bounded, $\left\{T(t)^{n} y_{n}\right\}_{t \geq 0}$ is uniformly bounded, $J$ is a weakly sequential continuous duality mapping, and $\alpha_{n} \in(a, b)$ that

$$
\begin{aligned}
\alpha_{n}[1 & \left.-\alpha_{n}(1+L)\right]\left\|x_{n}-T^{n}(t) x_{n}\right\|^{2} \\
\leq & \left\langle J\left(x_{n}-y_{n}\right)-J\left(x_{n+1}-y_{n}\right),\left(y_{n}-T^{n}(t) y_{n}\right)\right\rangle \\
& +\left(\mu_{n} M^{\prime \prime}(\operatorname{diam} C)+v_{n}\right), \\
\leq & \left\|J\left(x_{n}-y_{n}\right)-J\left(x_{n+1}-y_{n}\right)\right\|\left\|\left(y_{n}-T^{n}(t) y_{n}\right)\right\| \\
& +\left(\mu_{n} M^{*}(\operatorname{diam} C)+v_{n}\right) \rightarrow 0, \quad n \rightarrow \infty .
\end{aligned}
$$

So, $\left\|x_{n}-T^{n}(t) x_{n}\right\| \rightarrow 0$ as $n \rightarrow \infty$. In addition,

$$
\begin{aligned}
\| x_{n}- & T(t) x_{n} \| \\
\leq & \left\|x_{n+1}-x_{n}\right\|+\left\|T^{n+1}(t) x_{n+1}-x_{n+1}\right\| \\
\quad & \quad\left\|T^{n+1}(t) x_{n}-T^{n+1}(t) x_{n+1}\right\|+\left\|T(t) x_{n}-T^{n+1}(t) x_{n}\right\| \\
\leq & (L+1)\left\|x_{n+1}-x_{n}\right\|+\left\|T^{n+1}(t) x_{n+1}-x_{n+1}\right\|+L\left\|x_{n}-T^{n}(t) x_{n}\right\| .
\end{aligned}
$$

So, $\left\|x_{n}-T(t) x_{n}\right\| \rightarrow 0$ as $n \rightarrow \infty$.

(VI) Finally, we prove $x_{n} \rightarrow P_{F(T)} x$ as $n \rightarrow \infty$.

Let $x_{n_{k}}$ be a subsequence of $x_{n_{k}}$ such that $x_{n_{k}} \rightarrow \hat{x} \in C$, then by Theorem 3.1, we have $\hat{x} \in F(T)$. We let $\omega \in P_{F(T)} x$. For any $n \in \mathbb{N}, x_{n+1}=P_{H_{n} \cap W_{n}}$ and $\omega \in P_{F(T)} x \subset H_{n} \cap W_{n}$, so we get $\phi\left(x_{n+1}\right) \leq \phi(\omega, x)$.

On the other hand, from the weak lower semicontinuity of the norm, we have

$$
\begin{aligned}
\phi(\hat{x}, x) & =\|\hat{x}\|^{2}-2\langle\hat{x}, J x\rangle+\|x\|^{2} \\
& \leq \liminf _{n \rightarrow \infty}\left(\left\|x_{n_{k}}\right\|^{2}-2\left\langle\left\|x_{n_{k}}\right\|^{2}, J x\right\rangle+\|x\|^{2}\right) \\
& =\liminf _{n \rightarrow \infty} \phi\left(x_{n_{k}}, x\right) \\
& \leq \limsup _{n \rightarrow \infty} \phi\left(x_{n_{k}}, x\right) \\
& \leq \phi(\omega, x) .
\end{aligned}
$$

From the definition of $P_{F(T)} x$, we obtain $\hat{x}=\omega$ and hence $\lim \sup _{n \rightarrow \infty} \phi\left(x_{n_{k}}, x\right)=\phi(\omega, x)$. So, we have $\lim \sup _{k \rightarrow \infty}\left\|x_{n_{k}}\right\|=\|\omega\|$. Using the Kadec-Klee property of $E$, we obtain that $x_{n_{k}}$ converges strongly to $P_{F(T)} x$. Since $x_{n_{k}}$ is an arbitrary weakly convergent sequence of $x_{n}$, we can conclude that $x_{n}$ converges strongly to $P_{F(T)} x$. This completes the proof of Theorem 3.2 . 
Remark 3.1 Theorem 7 extends the main results of Zhou [8, 9], improves the results of Yang [17] and many others.

Remark 3.2 It is significant to remove the convexity of the duality mapping $J$ and the total asymptotically pseudocontractive semigroup in Theorem 7 of this article.

\section{Competing interests}

The authors declare that they have no competing interests.

\section{Authors' information}

All the authors contributed equally to the writing of the present article. And they also read and approved the final manuscript.

\section{Acknowledgements}

This work was supported by Scientific Research Fund Project of SiChuan Provincial Education Department (No. 11 ZA172 and No. 12ZB345).

Received: 23 August 2012 Accepted: 7 November 2012 Published: 28 November 2012

\section{References}

1. O'Regan, D, Shahzad, N: Krasnoselskii's fixed point theorem for general classes of maps. Adv. Fixed Point Theory 2, 248-257 (2012)

2. Ye, J, Huang, J: Strong convergence theorems for fixed point problems and generalized equilibrium problems of three relatively quasi-nonexpansive mappings in Banach space. J. Math. Comput. Sci. 1, 1-18 (2011)

3. Lau, AT, Takahashi, W: Invariant submeans and semigroups of nonexpansive mappings on Banach spaces with normal structure. J. Funct. Anal. 142, 79-88 (1996)

4. Cho, SY, Kang, SM: Approximation of fixed points pseudocontraction semigroups based on a viscosity iterative process. Appl. Math. Lett. 24, 224-228 (2011)

5. Xu, HK: A strong convergence theorem for contraction semigroups in Banach spaces. Bull. Aust. Math. Soc. 72, 371-379 (2005)

6. Chang, SS: Some results for asymptotically pseudo-contractive mappings and asymptotically nonexpansive mappings. Proc. Am. Math. Soc. 129, 845-853 (2001)

7. Osilike, MO, Akuchu, BG: Common fixed points of a finite family of asymptotically pseudocontractive mappings. Fixed Point Theory Appl. 2, 81-88 (2004)

8. Zhou, H: Demiclosedness principle with applications for asymptotically pseudo-contractions in Hilbert spaces. Nonlinear Anal., Theory Methods Appl. 70, 3140-3145 (2009)

9. Wang, Y-H, Xia, Y-H: Strong convergence for asymptotically pseudocontractions with the demiclosedness principle in Banach spaces. Fixed Point Theory Appl. 2012, 45 (2012). doi:10.1186/1687-1812-2012-45

10. Qin, X, Kim, JK, Wang, T: On the convergence of implicit iterative processes for asymptotically pseudocontractive mappings in the intermediate sense. Abstr. Appl. Anal. 2011, Article ID 468716 (2011)

11. Zhang, S: Convergence theorem of common fixed points for Lipschitzian pseudo-contraction semi-groups in Banach spaces. Appl. Math. Mech. (English Ed.) 30, 145-152 (2009)

12. Chidume, CE: Strong convergence theorems for fixed points of asymptotically pseudocontractive semi-groups. J. Math. Anal. Appl. 296, 410-421 (2004)

13. Alber, Yl, Chidume, CE, Zegeye, H: Approximating fixed point of total asymptotically nonexpansive mappings. Fixed Point Theory Appl. 2006, Article ID 10673 (2006)

14. Qin, X, Cho, SY, Kang, SM: A weak convergence theorem for total asymptotically pseudocontractive mappings in Hilbert spaces. Fixed Point Theory Appl. 2011, Article ID 859795 (2011). doi:10.1155/2011/859795

15. Alber, Yl: Metric and generalized projection operators in Banach spaces: properties and applications. In: Kartosator, AG (ed.) Theory and Applications of Nonlinear Operators of Accretive and Monotone Type, pp. 15-50. Dekker, New York (1996)

16. Kamimura, S, Takahashi, W: Strong convergence of a proximal-type algorithm in a Banach space. SIAM J. Optim. 13, 938-945 (2002)

17. Yang, L, Zhao, FH: Large strong convergence theorems for total asymptotically strict pseudocontractive semigroup in Banach spaces. Fixed Point Theory Appl. 2012, 24 (2012). doi:10.1186/1687-1812-2012-24

doi:10.1186/1687-1812-2012-216

Cite this article as: Wang and Quan: Strong convergence for total asymptotically pseudocontractive semigroups in

Banach spaces. Fixed Point Theory and Applications 2012 2012:216. 